Trauma or Drama: A Predictive Processing Perspective on the Continuum of Stress

Valery Krupnik

Author Note

Valery Krupnik, Department of Mental Health, Naval Hospital Camp Pendleton.

valery.y.krupnik.civ@mail.mil 


\begin{abstract}
The notion of trauma has been liberally used both in clinical literature and general discourse. However, no consensus exists on its exact meaning and definition. Whereas traditionally trauma has been mostly associated with criterion A of acute and post-traumatic stress disorders as defined in the Diagnostic and Statistical Manual of Mental Disorders, many researchers find this definition too constraining and not accounting for the complexity and many aspects of psychological trauma. This touched off a quest for a broader more accommodating trauma concept, and a dimensional view of trauma has been suggested with PTSD as its extreme manifestation. The dimensional view also has its detractors arguing that a "conceptual bracket creep” may undermine the category's utility. Both categorical and dimensional views mostly rely on trauma's clinical phenomenology and lack a unified theoretical basis. In an attempt to reconcile this contradiction, a hybrid categorical-dimensional model of trauma based on the general theory of stress has been recently proposed (Krupnik, 2019). In this report, we explore the categorical boundary of the trauma concept, as posited by the model, within the predictive processing framework (PPF). We integrate the PPF view with the theory of stress. In conclusion, we briefly discuss how the proposed model of trauma may guide clinical practice.
\end{abstract}

Keywords: trauma, allostasis, stress response, predictive processing, precision-weighting. 


\section{Trauma or Drama: A Predictive Processing Perspective on the Continuum of Stress}

The construct of trauma has been a subject of ongoing debate. In part, the difficulty to establish a consensus lies in the different facets of this concept that need to be reconciled and integrated. Trauma can refer to an event, experience, and symptoms, blurring the line between its cultural and medical meanings (Summerfield, 2001). Traditionally, there have been two trends in defining trauma. One is related to criterion A for acute and post-traumatic stress disorders in the consecutive issues of the Diagnostic and Statistical Manual of Mental Disorders (Diagnostic and Statistical Manual of Mental Disorder, 5th ed.; DSM-5; American Psychiatric Association, 2013), which in DSM-5 states "Exposure to actual or threatened death, serious injury, or sexual violence in one (or more) of the following ways...” (DSM-5, p 271). This criterion establishes a severity threshold in search of a categorical boundary (Weathers \& Keane 2007). Such a boundary is crucial for operationalizing trauma for research and practice since a category is only as useful as well it determines its boundaries. McNally notes that "conceptual bracket creep” carries the danger of trivializing trauma by eroding its singularity (McNally 2009). The need to distinguish between trauma and adversity has been demonstrated in research on childhood adversity and trauma (e.g. McLaughlin 2016, McCrory, De Brito, and Viding 2010). This body of research shows that different adverse childhood experiences lead to qualitatively different pathologies and that lumping them together under the category of trauma may be misleading.

Significant progress in understanding and treating PTSD, so that PTSD has become one of the most responsive to psychotherapy disorders (Bradley et al. 2005), has likely been assisted by the narrow DSM definition of trauma. However, its limitations have also been noted. One concerns type I and type II trauma and refers to the distinction between a singular traumatic 
event and cumulative trauma resulting from repeated (e.g. childhood) abuse (Terr 1991). Another is that PTSD diagnostic criteria do not capture developmental trauma, and developmental trauma disorder has been suggested as a separate category to be included in the DSM (Van der Kolk 2005). This suggests making trauma a more inclusive concept accommodating a wider scope of adverse experiences.

Whereas a more inclusive concept of trauma is likely to produce the very conceptual bracket creep that McNally warned against, some researchers have pursued this path developing a dimensional category of trauma. For example, the notion of PTSD as the highest degree of the normal stress response has been supported by a taxometric analysis of PTSD’s latent structure (Ruscio, Ruscio, \& Keane 2002, Broman-Fulks et al. 2009). Likewise, researchers of posttraumatic growth propose a dimensional view of adversity, where trauma is considered its highest degree (Seery, Holman, \& Silver 2010).

The dimensional view has been taken to its logical end by completely erasing the boundary between trauma and adversity as exemplified by the notion of a continuum from "small trauma” to "big trauma” (Shapiro 2017) and a fitting definition of trauma as "any event that has had a lasting negative effect upon self and psyche” (Shapiro 2017, p 39). The drive for hyperinclusivity of the trauma concept appears especially prevalent in the clinical realm, e.g. "There is more to trauma than PTSD” (Shapiro 2010, p. 11) or “It can perhaps be conjectured that unresolved trauma is responsible for a majority of the illnesses of modern mankind” (Levine 2010, p. 184). A practical corollary to the hyper-inclusive trauma concept is that trauma- and adversity-focused treatments become interchangeable. Indeed, cognitive-behavioral therapy (CBT) has been adapted to trauma treatment as trauma-focused CBT (Cohen, Mannarino, and Deblinger 2017) or CPT (Resick and Schnicke 1993), and, on the other hand, eye movement 
desensitization and reprocessing (EMDR), initially developed specifically for trauma, has since been adapted to a wide range of psychiatric and somatic conditions (Shapiro 2009).

In a recent attempt to reconcile the dimensional and categorical views of trauma, a hybrid categorical-dimensional model of trauma was presented in the context of the general theory of stress (Krupnik 2019), where trauma was defined as a particular kind of stress response. In this report, we build upon this model to further specify the properties of the traumatic stress response (TSR) in the predictive processing framework (PPF).

\section{Trauma as a Stress Response}

In the hybrid categorical-dimensional model, trauma is defined not as an event but the subjective experience of stress, i.e. stress response. The stress continuum is determined by two axes (Fig 1a): severity of stressors and strength of self-regulatory functions; their ratio determines the nature of stress response, i.e. its place on the continuum (Krupnik 2019). The continuum is divided into three categories (Fig 1a): normative stress response (NSR), pathogenic stress response (PSR), and traumatic stress response (TSR).

In NSR, the organism adapts to the stressors by returning to the optimal functional state, and no pathology ensues. In case of failure to maintain the optimal functionality, the organism undergoes allostasis (Sterling \& Eyer 1988, McEwen \& Wingfield 2003) to a new, sub-optimal, homeostatic state which can, therefore, be considered pathological. Allostasis can proceed either through PSR, where allostatic type 2 overload results in transition to a new homeostatic state with self-regulatory functions relatively intact, or through TSR, where allostatic type 1 overload triggers a breakdown of self-regulatory functions and an emergency response (for the difference 
between type 1 and 2 allostasis see e.g. (McEwen \& Wingfield 2003). ${ }^{1}$ A corresponding working definition of trauma was proposed (Krupnik, 2019, p. 259):

To be considered traumatic, a stress response to an event must meet a necessary condition that the event be outside of the person's normative life experience, and a sufficient condition that the response include a breakdown of self-regulatory functions.

The main feature distinguishing TSR from PSR was identified as a breakdown of selfregulatory functions. Neurological markers of such breakdown were hypothesized to associate with a malfunction of the default mode network (Raichle 2015) and clinical markers to largely overlap with PTSD symptoms (DSM-5). In the following sections, we explore the PSR/TSR difference in the predictive processing framework to further elucidate the functional basis of this distinction.

\section{Psychopathology in the Predictive Processing framework (PPF)}

\section{The predictive brain}

In a late paradigmatic shift, brain is no longer viewed as a passive receiver and processor of information. Instead, it is thought of as a predictive coding machine operating by rules of Bayesian inferential statistics (Clark 2013b, Friston 2010). In short, brain continuously runs a generative model of the external (the world) and internal (the body) environment. The model predicts the incoming sensory stimuli which reflect the states of the environment, by inferring

\footnotetext{
${ }^{1}$ Of note, the proposed categories of stress response are similar to McEwen's model which distinguishes "good," 'tolerable," and "toxic" stress (McEwen and Gianaros 2011), although the model is based on a different theoretical premise.
} 
their causes. These inferences are called priors or prior beliefs (or predictions) and are thought of as probability distribution functions representing prior learning. Prior beliefs are understood here in a broad sense, where they can reside at any level of information processing from unconscious expectancy to the declarative abstract thought. When sensory input does not match the prior, a prediction error (PE) is generated. PE's function is to update the brain's generative model, which happens through resolving/suppressing the PE. This can happen in two ways through a) posterior learning by adjusting the model's prior to match the input, or b) active inference by adjusting the organism's properties and/or behavior so that it controls the sensory input ('samples the environment') in a way that matches the model's prior. Through iterative cycles of perception-action brain directs the organism's behavior to selectively seek and gate sensory information to fulfill its predictions. An example of active inference is mammalian infants’ prediction that sucking on an object delivers a pleasurable sensation, making it possible to 'fool' them with a pacifier.

More recently, PPF has been high-powered by the free-energy principle (Friston 2010, Friston, Kilner, and Harrison 2006). The free-energy principle (FEP) provides a causal link between the mechanics of PPF and the teleological evolutionary frame. Being a product of evolution, the meta-purpose of PP is adaptation to the environment by anticipating its demands and by maintaining an optimal structural organization to meet them. Accordingly, FEP postulates that an organism's generative model is continuously increasing its accuracy through minimizing its variational free-energy (informational entropy). Variational free-energy is defined as the upper limit on surprisal or uncertainty about the brain’s sensory states. It is also related to structural entropy, providing a causal link to the second law of thermodynamics and, in turn, to the universal principle of self-organizing systems, which states that they self-organize by 
minimizing their entropy (Ashby 1991). Thus, FEP has been suggested as a universal theory of brain, where all brain functions can ultimately be traced to minimizing its free-energy (Friston 2010).

In order to run an accurate generative model, the brain has to optimally weight its priors against PEs, which is known as precision estimate or weighting (Friston 2009, Clark 2013a). Both priors and PEs are defined as probability distribution functions, whose inverse variance is called precision, i.e. high variance corresponds to low precision and vice versa. If the priors are too rigid (hyper-precise) they may be refractory to PE and fail to update according to the changing environment, rendering the generative model insensitive. On the other hand, too malleable priors (low precision) may lead to an unstable model, hypersensitive to environmental contingencies and thus lacking in predictive power. Precision-weighting is thought to be mediated by neuromodulatory control of the synaptic gain of PE units (Friston et al. 2014). Imbalance of precision-weighting with over- or under-weighted PE may result in false/inaccurate inference.

\section{Psychopathology as false inference}

Considering the free-energy principle a unified brain theory implies that malfunction of predictive processing could be viewed as the universal etiology of psychopathology (Friston et al. 2014). Indeed, a growing number of psychiatric conditions have been conceptualized in PPF, including psychosis (Powers, Mathys, \& Corlett 2017, Adams et al. 2013), depression (Badcock et al. 2017, Barrett, Quigley, and Hamilton 2016), anxiety disorders (Paulus, Feinstein, and Khalsa 2019), disorders of personality (Moutoussis et al. 2014), autism (Lawson, Rees, \& Friston 2014), functional neurological disorders (Edwards et al. 2012), ADHD (Dołęga 2018). 
Malfunction of PP can happen in different inter-related domains: perceptive inference including extero- and interoception and active inference including motor and visceromotor action, respectively. Suboptimal precision-weighting leading to false inference has been identified as central to psychiatric etiology (Friston et al. 2014). For instance, false proprioceptive inference has been implicated in motor movement disorders (Edwards et al. 2012), whereas false exteroceptive inference - in psychosis (Adams et al. 2013, Powers, Mathys, \& Corlett 2017). False interoceptive inference has mostly been implicated in mood/affective disorders (Paulus, Feinstein, \& Khalsa 2019).

\section{Maladaptive stress response as false inference}

Psychopathology has also been conceptualized as inadequate stress response, which is widely known as the diathesis-stress model. The model posits that the combination of stress severity and vulnerability to stress determines whether stress results in resilience or pathology (Monroe \& Simons 1991). This presumes a continuum of stress response from resilience to pathology, similar to the one in Figure 1a. In allostasis terms, healthy vs pathologic stress response depends on how adaptively the organism negotiates allostatic load by adjusting its homeostatic states within the physiological range (Sterling 2012). Self-regulation is believed to mediate such adaptability.

In the last decade, the theory of allostasis has been integrated into PPF. Survival in a changing environment is the ultimate goal of behavior, therefore, allostasis is the brain's evolutionary purpose and primary function (Sterling, 2012). Accordingly, allostasis is understood as an organism's ability to adjust its internal state to anticipated future challenges (e.g. increasing the blood pressure in preparation for standing up). Such anticipation is encoded 
by the brain's generative model, specifically, its interoceptive inference. Thus, interoceptive inference has been proposed as the mechanism of allostasis (Peters, McEwen, \& Friston 2017, Barrett \& Simmons 2015).

Interoceptive inference is underwritten, as discussed above, by the free-energy principle stipulating that brain's ultimate function is to minimize the uncertainty about its sensory states (internal states in case of interoception). Then, the meaning of an adaptive stress response (successful allostasis) is an accurate prediction of internal states that optimally accommodate the future challenge.

\section{Predictive Processing Account of Trauma}

PPF has recently been applied to trauma (Wilkinson, Dodgson, \& Meares 2017, Linson \& Friston 2019), where different manifestations of PTSD are regarded as a trauma-induced malfunction of the brain's generative model. For example, dissociation is thought to result from the fragmentation of the generative model, where the model of the self is disconnected from the model of the traumatic event. Consequently, the self-model fails to be updated by the ascending interoceptive prediction errors, making the trauma felt as happening not to oneself (Wilkinson, Dodgson, \& Meares 2017). As a result, the negative affect is mitigated during a traumatic event, which may be adaptive as a peri-traumatic response but becomes maladaptive long-term, making dissociation one of PTSD symptoms (DSM-5). On the other hand, the post-traumatic model of the world is believed to rely on hyper-precise predictions of threat that do not require sensory confirmation for triggering a metabolic 'fight or flight' response (Linson \& Friston 2019). We think that the hypothesized insensitivity (under-weighting) to PE is an essential property of traumatic stress response and will expound it further below. 


\section{Traumatic stress response (TSR) as type 1 allostasis}

Emergency response to stress is one of the features of allostatic overload type 1 (McEwen \& Wingfield 2003). This emergency quality links type 1 allostasis to the necessary condition of TSR in the above definition of trauma, i.e. "the event is outside of the person's normative life experience.” Outside its normative range of experience, the organism’s generative model receives a sensory input that it predicts as highly improbable which generates a hyper-precise prediction error. This is likely to trigger a dramatic increase in the model's uncertainty/free energy. In order to compensate for it, just as dramatic a response may be required. The two available pathways, as mentioned above, are acting on the environment according to active inference or updating the priors through posterior learning, i.e. adjusting the perceptive inference. In a traumatic event, action may not be available, which can render the event ever so traumatizing. Then, the remaining option is an abrupt overhaul of the model's network of priors, often leading to dysregulation within the model, e.g. dissociation or psychosis (Wilkinson, Dodgson, \& Meares 2017). Such dysregulation corresponds to "breakdown of self-regulatory functions” suggested as the sufficient condition and a defining feature of TSR (Krupnik 2019).

Type 2 overload, on the contrary, is associated with a lower intensity chronic challenge to the generative model (McEwen \& Wingfield 2003), which would be associated with lower precision PEs that may not be out of range of its priors. This may present an opportunity for a gradual response combining a compensatory active inference with a measured adjustment of perceptive inference. Such a response may still prove suboptimal and entail pathology (PSR), since the allostatic load may still 'lock' the organism in a limited/forced range of stress response (Juster, McEwen, \& Lupien 2010). We suggest that lack of pathology differentiate NSR from 
both PSR and TSR, whereas the nature of PP malfunction differentiates PSR from TSR (Fig 1). Below, we specify the kind of malfunction we hypothesize to differentiate TSR from PSR. ${ }^{2}$

\section{TSR as dysregulation of precision weighting}

We hypothesize that in TSR the generative model of the world undergoes a drastic recalibration of its predictions (exteroceptive priors). As a result, the model generates a set of hyper-precise predictions of the world's dangerousness to accommodate the traumatic experience in a way that suppresses the prediction error thus minimizing the model's free energy (Fig 2b). Such recalibration results in a biased exteroceptive inference about the world as inherently threatening, which mindset is a hallmark manifestation of PTSD (DSM-5) including symptoms of hypervigilance and unrealistic negative beliefs about the world. Hyper-precise 'threat' priors will result in relatively underweighted exteroceptive PEs (ePE). We suggest that in PPF precision-weighting functions as a mechanism of allostasis, where the ratio between a prior and the PE precisions determines the current homeostatic setpoint for the prior, i.e. its mean and variance. Under allostatic load, these parameters change in a way that minimizes the model's free energy.

Trauma-induced exteroceptive 'blindness’ is expected to be trauma-specific; however, it may generalize to unrelated or loosely related sensory information. For example, PTSD patients show hyper-activation of the amygdala in response to both trauma-specific and neutral images. Moreover, this hyper-reactivity is observed even without recognition of presented stimuli,

\footnotetext{
${ }^{2}$ We want to emphasize that the proposed malfunction need not be limited to TSR but may be found in other pathologies; it only becomes a trauma attribute when caused by a stressor outside the organism's normative range of experience.
} 
indicating that it is likely due to the top-down, i.e. prediction-driven, information processing (Hendler et al. 2003).

Peritraumatic reaction to type 1 allostatic overload is associated with an extreme visceromotor response commonly known as ‘fight, flight, or freeze' (Barlow 2004) which can be regarded as a visceromotor active inference about a life-threatening situation (Hutchinson \& Barrett 2019). If so, such inference is expected to return to its normative range once the threat is over. However, if the mind is under control of hyper-precise exteroceptive 'threat' priors, the threat never ceases (Wilkinson, Dodgson, \& Meares 2017). Consequently, to minimize uncertainty, interoceptive inference needs to match the exteroceptive one by, too, becoming hyper-precise in predicting an emergency stress response (Fig 2b). This would lead to selective underweighting of interoceptive PE (iPE)s and, consequently, 'lock' the mind in a selfperpetuating cycle of TSR, where hyper-precise exteroceptive and interoceptive priors feed into each other. Indeed, such a hallmark PTSD symptom as hypervigilance (DSM-5) can be seen as both an active inference (hypervigilant behaviors) 'fulfilling' the hyper-precise exteroceptive 'threat' priors and interoceptive active inference (visceromotor manifestations) 'fulfilling' the hyper-precise interoceptive predictions of physiological emergency response (Fig 2b).

We suggest that the described dysregulation of precision-weighting in TSR differentiates it from PSR (Fig 2a). PSR results from allostatic overload type 2, where the organism faces no acute survival/reproductive threat but is under environmental pressure that may drive it into a suboptimal homeostatic range. This does not necessitate an abrupt recalibration of the priors into hyper-precise 'threat' predictions. Instead, the generative model may decrease the priors' precision to better accommodate the higher range of error and minimize the uncertainty in that way. This will result in a relative increase of ePE's precision and their overweighting in the 
service of allostasis (Fig 2a). As in TSR, we expect such overweighting to be stressor-specific to a degree but allow for its generalization. For example, in generalized anxiety disorder, people react with anxiety to a large variety of external and internal stimuli with a higher share of miscellaneous items compared to controls (Roemer, Molina, \& Borkovec 1997).

Overweighting of ePE presents a challenge for the interoceptive inference. In an inherently uncertain world (high precision ePE) it is difficult to predict an appropriate visceromotor response. This challenge was aptly expressed in application to depression, "major depression occurs when the brain is certain that it will encounter an uncertain environment, i.e. the world is inherently volatile, capricious, unpredictable and uncontrollable” (Clark, Watson, \& Friston 2018, p 2278). Two opposite strategies can be envisioned for interoceptive inference to 'keep up.' One is to 'relax' the precision of its priors and accommodate/overweight the iPE. This would lead to the organism's over-reaction to its “noisy" internal sensation as was previously hypothesized (Barrett, Quigley, and Hamilton 2016, Clark, Watson, \& Friston 2018). The other would be to increase interoceptive priors' precision, making the generative model relatively insensitive to internal sensations (and iPE) and thus ever ready for allostatic overload as in TSR. The latter strategy was also recently hypothesized (Krupnik 2020). Indeed, chronic hyperarousal has been noted in anxiety and depression (Greaves-Lord et al. 2007). Hence, interoceptive inference can compensate for the overweighting of ePE by underweighting iPE (Fig 2a). Accordingly, the proposed model of the stress continuum (Krupnik 2019) can be developed into a model of stress response continuum (Fig 1b). Since the stress continuum model defines stress as the subjective experience of stressors, it follows that a stress response continuum model defines stress response as the organism's (and its brain's) response to that experience, or allostasis. Then the axes determining the stress continuum (Fig 1a) can be transmuted to frame 
the stress response continuum. The strength of self-regulatory functions in allostasis terms denotes the range of adaptive physiological reactions to stress or, in PP interoceptive terms, the predicted range of allostasis, hence this axis can be transmuted into 'range of allostasis.' Likewise, in allostasis terms, severity of stressors translates into allostatic load which becomes the other axis of the continuum (Fig 1b).

In order to elaborate the described model in greater clinical detail, below, we compare TSR to PSR, using examples of their prototypical clinical conditions, PTSD and depression, respectively. A detailed description of the PSR model of depression can be found elsewhere (Krupnik 2020).

\section{PTSD and Depression as Different kinds of Stress Response}

Despite their high comorbidity (Bleich et al. 1997) depression and PTSD are clinically distinct. One of the hallmarks of PTSD is avoidance (DSM-5), where patients avoid reminders, thoughts, and feelings associated with the traumatic event. Depressed people, on the contrary, tend to engage in rumination, i.e. prolonged reminiscing and brooding about their past and present misfortune (Nolen-Hoeksema, Wisco, \& Lyubomirsky 2008). This contrast is further underscored by the difference between neurological signatures of depressive rumination and recall of traumatic memories. The latter is associated with deactivation of the medial prefrontal cortex in PTSD patients (Lanius et al. 2002), whereas the former shows increased activity in this area (Nolen-Hoeksema, Wisco, \& Lyubomirsky 2008).

The significance of these differences for the stress response model is several-fold. The medial prefrontal cortex is part of the default mode network which plays a central role in selfregulation (Raichle 2015). Its inhibition during the processing of disturbing information in PTSD 
is consistent with the malfunction of self-regulation proposed as the main feature of TSR in the definition of trauma (Krupnik 2019). Breakdown of self-regulation, including peri- and posttraumatic dissociation, has been long associated with trauma and PTSD (van der Kolk \& van der Hart 1989). Neither impaired self-regulation nor dissociation has been noted as core features of depression, moreover, the adaptive value of depressive stress response including rumination has been highlighted by some researchers (Andrews \& Thompson 2009, Nesse 2000, Gilbert 2006).

Linson \& Friston (2019) suggest that a traumatized generative model is characterized by decoupling of top-down priors from the bottom-up sensory information processing, which makes the organism react to the sensory cues according to its 'threat' priors. This decoupling is seen as the mechanism of impaired reality testing observed in such PTSD symptoms as hypervigilance and increased startle response (to auditory stimuli, in particular) and implies selective underweighting of ePEs. These reactions along with experiential avoidance can be interpreted as an aberrant active inference 'fulfilling' the hyper-precise threat priors (Fig 2b).

On the interoceptive side, PTSD is characterized by chronic hyperarousal (DSM-5) which in PPF can be regarded as active interoceptive inference meant to minimize the uncertainty of a generative model that over-predicts threat. Selective underweighting of iPE helps sustain the hyper-precise ‘allostasis’ priors. Thus, PTSD generative model is defined by a combination of 'runaway’ exteroceptive ‘threat’ and interoceptive ‘allostasis’ priors (Fig 2b), which makes it prone to recreating the virtual traumatic experience when triggered. This may explain 'reexperiencing,' the most specific PTSD symptom (DSM-5). Noteworthy, such recreating often happens in night dreams, when there is little to no sensory input and, consequently, no ePE.

There are several accounts of depression in PPF (Barrett, Quigley, \& Hamilton 2016, Badcock et al. 2017) (Chekroud 2015, Fabry 2019, Kube et al. 2019). They point to a 
commonality between depressive and PTSD generative models. Both have been characterized by hyper-precise interoceptive allostasis priors and underweighted PE (Fig 2), which maintain the organism in a state of allostatic overload in anticipation of an inimical/dangerous environment. Such a state has been dubbed a “locked-in” brain (Barrett, Quigley, \& Hamilton 2016) or selective interoceptive blindness (Krupnik 2020). In depression, it manifests in increased anxiety and hyperarousal (e.g. insomnia) instead of hypervigilance. There have also been suggestions that depression is associated with overweighted iPE (Clark, Watson, \& Friston 2018), and indeed hypoarousal and lethargy are also featured in depression (Greaves-Lord et al. 2007). The dynamic model of depression resolves this apparent contradiction by taking into account different phases (with different manifestations) of depressive stress response (Krupnik 2014, Krupnik 2020).

Where depressive and PTSD generative models differ is exteroceptive inference (Fig 2). Without the need for an abrupt re-calibration of exteroceptive priors (as in trauma) the organism can use the alternative strategy of decreasing the precision of its exteroceptive priors to better accommodate the depressogenic ePE and thus minimize the model's uncertainty. The ensuing overweighting of exteroceptive PE may explain such clinical manifestations of depression as rumination about environmental challenges as opposed to avoidance, psychomotor retardation and indecisiveness as opposed to increased startle response (DSM-5).

In accord with the highlighted differences between TSR and PRS generative models (Fig 2), we suggest to specify the stress continuum (Fig 1b) and update the earlier (Krupnik 2019) definition of trauma in the following way: 
To be considered traumatic, a stress response to an event must meet the necessary condition that the event be outside of the person's normative life experience, causing an abrupt recalibration of exteroceptive priors, and the sufficient condition that the response include a breakdown of self-regulatory functions manifested in a malfunction of predictive processing with selectively underweighted precision of exteroceptive and interoceptive prediction errors.

\section{Empirical Evidence}

Whereas the described model of TSR (Fig 1, 2) is speculative and has not been directly tested, much of empirical knowledge about trauma appears consistent with it. The evidence that trauma and PTSD, in particular, are associated with false inference, where hyper-precise threat priors skew the perception (sometimes to the point of flashback and hallucination) has been reviewed before (Wilkinson, Dodgson, \& Meares 2017). Likewise, false interoceptive inference in PTSD has been extensively discussed (Linson \& Friston 2019). Here, we want to highlight perhaps the most direct indications of impaired predictive processing in trauma. Two related studies explored the encoding of prediction error in trauma (Ross et al. 2018, Lenow et al. 2014). In a reinforcement learning task, the authors demonstrated a decreased PE encoding in the medial PFC/ventral striatum network and anterior insula in people with PTSD (Ross et al. 2018). Low anterior insula PE encoding in traumatized individuals was also observed in a trust violation paradigm (Lenow et al. 2014). Medial PFC/ventral striatum network is responsible for assigning value to external information (Rushworth \& Behrens 2008), whereas anterior insula is considered the hub of interoception (Craig 2002). These data support our suggestion that a traumatized generative model is characterized by selective underweighting of extero- and interoceptive PE (Fig 2b). 
Depression, too, has been associated with low interoceptive sensory sensitivity, i.e. underweighted iPE. Phenomenologically, it is manifested in the emotional flatness of depressed people (Rottenberg, Gross, \& Gotlib 2005). Depressed patients show decreased activity in the insula during an interoceptive task (Avery et al. 2014). The impaired interoceptive accuracy in depression has been documented in a recent comprehensive review (Eggart et al. 2019). The cumulative findings show a correlation between low affectivity and low interoceptive accuracy. Interestingly, the largest interoceptive deficit is noted in moderate depression, whereas it normalizes in severe one. This seemingly paradoxical finding fits the dynamic PPF model of depression (Krupnik 2020) ${ }^{3}$.

The central claim of our model is that in contrast to underweighted ePEs in trauma, depression is associated with their selective overweighting. According to Badcock et al. (2017), it may result from suspended sensory attenuation due to impaired neuromodulation by dopamine and serotonin. The converging evidence of impaired ePE processing has recently been systemically reviewed (Kube et al. 2019). Specifically, depressed patients show increased lossrelated PE encoding in ventral striatum (Ubl et al. 2015). In contrast to PTSD, depressed patients appear to have an intact reward PE encoding in ventral striatum (Rutledge et al. 2017). Applying the work on prediction error dynamics (Kiverstein, Miller, \& Rietveld 2019) to depression, Fabry (2019) explores the hypothesis that the depressive generative model is caught in a cycle of overestimating the rate of PE minimization, leading to accumulation of a "larger-than-expected" (i.e. overweighted) PE.

In aggregate, the existing evidence points to the qualitative difference between neural dynamics in PTSD and depression. The suggested model (Fig 2) interprets this difference in PE

\footnotetext{
3 The dynamic model also accommodates the related paradox known as 'the dark room paradox' as it applies to depression (Fabry, 2019).
} 
processing terms, contrasting traumatic with pathogenic (Fig 1) stress response. More research is needed to further clarify the difference in PPF terms between PTSD and depression, specifically by direct comparison of respective patients in the same experimental paradigm.

\section{Discussion}

Perhaps the most significance of the suggested model of stress continuum (Fig 1) is in its practical application. Specifically, the different nature of TSR vs PSR implies that different therapeutic approaches may be efficacious in either case. This goes against the late trend toward universal psychotherapies. For example, cognitive-behavioral therapy (CBT) has been used and found efficacious for a plethora of mental conditions, and its various modifications have cropped up including trauma-focused CBT (Cohen, Mannarino, \& Deblinger 2017). The opposite tendency, i.e. toward specificity of psychosocial interventions for their target, also has its proponents (e.g. Parker, Roy, \& Eyers 2003).

The proposed model implies different therapeutic strategies for TSR and PSR. All efficacious therapies for PTSD have exposure at their core. This appears a straightforward approach since it allows for reshaping the patient's perceptual and active inference (including interoceptive) response by changing the external and internal context of traumatic imagery as well as behavioral reaction to it. On the other hand, using exposure for the treatment of depression may not provide significant benefits because unlike PTSD patients, depressed people do not avoid the negative material but self-expose to it through rumination (Nolen-Hoeksema, Wisco, \& Lyubomirsky 2008). Accordingly, it was proposed that instead of the negative material the dynamics of depressive response may be a better target for intervention (Krupnik 2014) and a 
corresponding modification of a trauma-focused therapy has been developed for depression (Krupnik 2018). 


\section{References}

American Psychiatric Association. (2013). Diagnostic and statistical manual of mental disorders (5th ed.). Arlington, VA: American Psychiatric Publishing

Adams, Rick, Klaas Stephan, Harriet Brown, Christopher Frith, and Karl Friston. 2013. "The Computational Anatomy of Psychosis." Frontiers in Psychiatry 4:47. doi: 10.3389/fpsyt.2013.00047.

Andrews, P. W., and J. A. Thompson. 2009. "The bright side of being blue: Depression as an adaptation for analyzing complex problems." Psychol Review 116 (3):620-54.

Ashby, W. Ross. 1991. "Principles of the Self-Organizing System." In Facets of Systems Science, 521-536. Boston, MA: Springer US.

Avery, Jason A., Wayne C. Drevets, Scott E. Moseman, Jerzy Bodurka, Joel C. Barcalow, and W. Kyle Simmons. 2014. "Major Depressive Disorder Is Associated With Abnormal Interoceptive Activity and Functional Connectivity in the Insula." Biological Psychiatry 76 (3):258-266. doi: https://doi.org/10.1016/j.biopsych.2013.11.027.

Badcock, Paul B, Christopher G Davey, Sarah Whittle, Nicholas B Allen, and Karl J Friston. 2017. "The Depressed Brain: An Evolutionary Systems Theory." Trends in Cognitive Sciences 21 (3):182-194. doi: https://doi.org/10.1016/j.tics.2017.01.005.

Barlow, David H. 2004. Anxiety and its disorders: The nature and treatment of anxiety and panic: Guilford press.

Barrett, Lisa Feldman, Karen S Quigley, and Paul Hamilton. 2016. "An active inference theory of allostasis and interoception in depression." Phil. Trans. R. Soc. B 371 (1708):1-17. doi: 10.1098/rstb.2016.0011. 
Barrett, Lisa Feldman, and W. Kyle Simmons. 2015. "Interoceptive predictions in the brain." Nature reviews. Neuroscience 16 (7):419-429. doi: 10.1038/nrn3950.

Bleich, Avi, Meni Koslowsky, Aliza Dolev, and Bernard Lerer. 1997. "Post-traumatic stress disorder and depression: An analysis of comorbidity." The British Journal of Psychiatry 170 (5):479-482. doi: 10.1192/bjp.170.5.479.

Bradley, Rebekah, Jamelle Greene, Eric Russ, Lissa Dutra, and Drew Westen. 2005. "A multidimensional meta-analysis of psychotherapy for PTSD." American journal of Psychiatry 162 (2):214-227. doi: 10.1176/appi.ajp.162.2.214

Broman-Fulks, Joshua J, Kenneth J Ruggiero, Bradley A Green, Daniel W Smith, Rochelle F Hanson, Dean G Kilpatrick, and Benjamin E Saunders. 2009. "The latent structure of posttraumatic stress disorder among adolescents." Journal of Traumatic Stress 22 (2):146-152. doi: 10.1002/jts.20399.

Chekroud, Adam M. 2015. "Unifying treatments for depression: an application of the Free Energy Principle." Frontiers in Psychology 6 (153). doi: 10.3389/fpsyg.2015.00153.

Clark, Andy. 2013a. "The many faces of precision (Replies to commentaries on "Whatever next? Neural prediction, situated agents, and the future of cognitive science”)." Frontiers in Psychology 4 (270). doi: 10.3389/fpsyg.2013.00270.

Clark, Andy. 2013b. "Whatever next? Predictive brains, situated agents, and the future of cognitive science." Behavioral and brain sciences 36 (3):181-204. doi: doi:10.1017/S0140525X12000477.

Clark, James E., Stuart Watson, and Karl J. Friston. 2018. "What is mood? A computational perspective." Psychological Medicine 48 (14):2277-2284. doi: 10.1017/S0033291718000430. 
Cohen, Judith A, Anthony P Mannarino, and Esther Deblinger. 2017. Treating trauma and traumatic grief in children and adolescents. second ed. New York: Guilford Publications.

Craig, AD. 2002. "How do you feel? Interoception: the sense of the physiological condition of the body." Nat Rev Neurosci 3 (8):655-66. doi: 10.1038/nrn894.

Dołęga, Krzysztof. 2018. "Commentary: M-Autonomy." Frontiers in Psychology 9:680. doi: 10.3389/fpsyg.2018.00680.

Edwards, Mark J., Rick A. Adams, Harriet Brown, Isabel Pareés, and Karl J. Friston. 2012. "A Bayesian account of 'hysteria’." Brain 135 (11):3495-3512. doi: 10.1093/brain/aws129.

Eggart, Michael, Andreas Lange, Martin J Binser, Silvia Queri, and Bruno MüllerOerlinghausen. 2019. "Major Depressive Disorder Is Associated with Impaired Interoceptive Accuracy: A Systematic Review." Brain sciences 9 (6):131. doi: 10.3390/brainsci9060131

Fabry, Regina E. 2019. "Into the dark room: a predictive processing account of major depressive disorder." Phenomenology and the Cognitive Sciences:1-20. doi: 10.1007/s11097-01909635-4.

Friston, Karl. 2009. "The free-energy principle: a rough guide to the brain?" Trends in cognitive sciences 13 (7):293-301. doi: https://doi.org/10.1016/j.tics.2009.04.005.

Friston, Karl. 2010. "The free-energy principle: a unified brain theory?" Nat Rev Neurosci 11 (2):127-138. doi: 10.1038/nrn2787.

Friston, Karl J, Klaas Enno Stephan, Read Montague, and Raymond J Dolan. 2014. "Computational psychiatry: the brain as a phantastic organ." The Lancet Psychiatry 1 (2):148-158. doi: https://doi.org/10.1016/S2215-0366(14)70275-5. 
Friston, Karl, James Kilner, and Lee Harrison. 2006. "A free energy principle for the brain." Journal of Physiology-Paris 100 (1):70-87. doi: https://doi.org/10.1016/j.jphysparis.2006.10.001.

Gilbert, P. 2006. "Evolution and depression: issues and implications." Psychol Med 36 (3):28797. doi: 10.1017/S0033291705006112.

Greaves-Lord, Kirstin, Robert F Ferdinand, Frouke EPL Sondeijker, Andrea Dietrich, Albertine J Oldehinkel, Judith GM Rosmalen, Johan Ormel, and Frank C Verhulst. 2007. "Testing the tripartite model in young adolescents: Is hyperarousal specific for anxiety and not depression?" Journal of Affective Disorders 102 (1-3):55-63. doi: 10.1016/j.jad.2006.12.009.

Hendler, Talma, Pia Rotshtein, Yaara Yeshurun, Tal Weizmann, Itamar Kahn, Dafna BenBashat, Rafael Malach, and Avi Bleich. 2003. "Sensing the invisible: differential sensitivity of visual cortex and amygdala to traumatic context." Neuroimage 19 (3):587600. doi: 10.1016/S1053-8119(03)00141-1.

Hutchinson, J. Benjamin, and Lisa Feldman Barrett. 2019. "The Power of Predictions: An Emerging Paradigm for Psychological Research." Current Directions in Psychological Science 28 (3):280-291. doi: 10.1177/0963721419831992.

Juster, Robert-Paul, Bruce S McEwen, and Sonia J Lupien. 2010. "Allostatic load biomarkers of chronic stress and impact on health and cognition." Neuroscience \& Biobehavioral Reviews 35 (1):2-16. doi: 10.1016/j.neubiorev.2009.10.002.

Kiverstein, Julian, Mark Miller, and Erik Rietveld. 2019. "The feeling of grip: novelty, error dynamics, and the predictive brain." Synthese 196 (7):2847-2869. doi: 10.1007/s11229017-1583-9. 
Krupnik, Valery. 2014. "A Novel Therapeutic Frame for Treating Depression in Group Treating Depression Downhill." SAGE Open 4 (1):1-12. doi: 10.1177/2158244014523793.

Krupnik, Valery. 2018. "Differential Effects of an Evolutionary-Based EMDR Therapy on Depression and Anxiety Symptoms: A Case Series Study." Journal of EMDR Practice and Research 12 (2):46-57. doi: 10. 1891/ 1933- 3196. 12. 2. 46.

Krupnik, Valery. 2019. "Trauma or adversity?" Traumatology 25 (4):256-261. doi: 10.1037/trm0000169.

Krupnik, Valery. 2020. " On a Path to Integration of the Theory and Practice of Depression: Evolution, Stress, and Predictive Processing." In Depression and Anxiety: Prevalence, Risk Factors and Treatment, 1-54, ed Shelley L. Becker, New York: Nova Science Publishers.

Kube, Tobias, Rainer Schwarting, Liron Rozenkrantz, Julia Anna Glombiewski, and Winfried Rief. 2019. "Distorted Cognitive Processes in Major Depression: A Predictive Processing Perspective." Biological Psychiatry. doi: 10.1016/j.biopsych.2019.07.017.

Lanius, Ruth A, Peter C Williamson, Kristine Boksman, Maria Densmore, Madhulika Gupta, Richard WJ Neufeld, Joseph S Gati, and Ravi S Menon. 2002. "Brain activation during script-driven imagery induced dissociative responses in PTSD: a functional magnetic resonance imaging investigation." Biological psychiatry 52 (4):305-311.

Lawson, Rebecca P., Geraint Rees, and Karl J. Friston. 2014. "An aberrant precision account of autism." Frontiers in Human Neuroscience 8 (302). doi: 10.3389/fnhum.2014.00302.

Lenow, Jennifer K, J Scott Steele, Sonet Smitherman, Clinton D Kilts, and Josh M Cisler. 2014. "Attenuated behavioral and brain responses to trust violations among assaulted 
adolescent girls." Psychiatry Research: Neuroimaging 223 (1):1-8. doi: 10.1016/j.pscychresns.2014.04.005.

Levine, Peter A. 2010. In an unspoken voice: How the body releases trauma and restores goodness. Berkeley: North Atlantic Books.

Linson, Adam, and Karl Friston. 2019. "Reframing PTSD for computational psychiatry with the active inference framework." Cognitive Neuropsychiatry 24 (5):347-368. doi: 10.1080/13546805.2019.1665994.

McCrory, Eamon, Stephane A De Brito, and Essi Viding. 2010. "Research review: the neurobiology and genetics of maltreatment and adversity." Journal of Child Psychology and Psychiatry 51 (10):1079-1095. doi: 10.1111/j.1469-7610.2010.02271.x.

McEwen, Bruce S, and Peter J Gianaros. 2011. "Stress-and allostasis-induced brain plasticity." Annual review of medicine 62:431-445. doi: 10.1146/annurev-med-052209-100430.

McEwen, Bruce S, and John C Wingfield. 2003. "The concept of allostasis in biology and biomedicine." Hormones and behavior 43 (1):2-15. doi: https://doi.org/10.1016/S0018506X(02)00024-7.

McLaughlin, Katie A. 2016. "Future directions in childhood adversity and youth psychopathology." Journal of Clinical Child \& Adolescent Psychology 45 (3):361-382. doi: 10.1080/15374416.2015.1110823

McNally, Richard J. 2009. "Can we fix PTSD in DSM-V?" Depression and anxiety 26 (7):597600. doi: 10.1002/da.20586.

Monroe, Scott M, and Anne D Simons. 1991. "Diathesis-stress theories in the context of life stress research: implications for the depressive disorders." Psychological bulletin 110 (3):406-425. doi: 10.1037/0033-2909.110.3.406. 
Moutoussis, Michael, Pasco Fearon, Wael El-Deredy, Raymond J. Dolan, and Karl J. Friston. 2014. "Bayesian inferences about the self (and others): A review." Consciousness and Cognition 25 (Supplement C):67-76. doi: https://doi.org/10.1016/j.concog.2014.01.009.

Nesse, R. M. 2000. "Is depression an adaptation?" Archives of General Psychiatry 57 (1):14-20.

Nolen-Hoeksema, Susan, Blair E Wisco, and Sonja Lyubomirsky. 2008. "Rethinking rumination." Perspectives on psychological science 3 (5):400-424. doi: 10.1111/j.17456924.2008.00088.x.

Parker, G., K. Roy, and K. Eyers. 2003. "Cognitive behavior therapy for depression? Choose horses for courses." Am J Psychiatry 160 (5):825-34.

Paulus, Martin P, Justin S Feinstein, and Sahib S Khalsa. 2019. "An active inference approach to interoceptive psychopathology." Annual review of clinical psychology 15:97-122. doi: 10.1146/annurev-clinpsy-050718-095617.

Peters, Achim, Bruce S. McEwen, and Karl Friston. 2017. "Uncertainty and stress: Why it causes diseases and how it is mastered by the brain." Progress in Neurobiology 156:164-188. doi: https://doi.org/10.1016/j.pneurobio.2017.05.004.

Powers, AR, C Mathys, and PR Corlett. 2017. "Pavlovian conditioning-induced hallucinations result from overweighting of perceptual priors." Science 357 (6351):596-600. doi: 10.1126/science.aan3458.

Raichle, Marcus E. 2015. "The brain's default mode network." Annual review of neuroscience 38:433-447. doi: 10.1146.

Resick, Patricia A, and Monica Schnicke. 1993. Cognitive processing therapy for rape victims: A treatment manual. Newbury Park, CA: Sage. 
Roemer, Lizabeth, Silvia Molina, and Thomas D. Borkovec. 1997. "An Investigation of Worry Content among Generally Anxious Individuals." The Journal of Nervous and Mental Disease 185 (5):314-319.

Ross, Marisa C, Jennifer K Lenow, Clinton D Kilts, and Josh M Cisler. 2018. "Altered neural encoding of prediction errors in assault-related posttraumatic stress disorder." Journal of psychiatric research 103:83-90. doi: 10.1016/j.jpsychires.2018.05.008.

Rottenberg, Jonathan, James J. Gross, and Ian H. Gotlib. 2005. "Emotion Context Insensitivity in Major Depressive Disorder." Journal of Abnormal Psychology 114 (4):627-639. doi: 10.1037/0021-843X.114.4.627.

Ruscio, Ayelet Meron, John Ruscio, and Terence M Keane. 2002. "The latent structure of posttraumatic stress disorder: A taxometric investigation of reactions to extreme stress." Journal of abnormal psychology 111 (2):290-301. doi: 10.1037//0021-843X.111.2.290.

Rushworth, Matthew FS, and Timothy EJ Behrens. 2008. "Choice, uncertainty and value in prefrontal and cingulate cortex." Nature neuroscience 11 (4):389-397. doi: 10.1038/nn2066.

Rutledge, Robb B., Michael Moutoussis, Peter Smittenaar, Peter Zeidman, Tanja Taylor, Louise Hrynkiewicz, Jordan Lam, Nikolina Skandali, Jenifer Z. Siegel, Olga T. Ousdal, Gita Prabhu, Peter Dayan, Peter Fonagy, and Raymond J. Dolan. 2017. "Association of Neural and Emotional Impacts of Reward Prediction Errors With Major Depression." JAMA Psychiatry 74 (8):790-797. doi: 10.1001/jamapsychiatry.2017.1713.

Seery, Mark D, E Alison Holman, and Roxane Cohen Silver. 2010. "Whatever does not kill us: cumulative lifetime adversity, vulnerability, and resilience." Journal of personality and social psychology 99 (6):1025-1041. doi: 10.1037/a0021344. 
Shapiro, F. 2017. Eye Movement Desensitization and Reprocessing (EMDR) Therapy: Basic principles, protocols and procedures. 3rd ed. New York: Guilford Press.

Shapiro, Robin. 2010. The trauma treatment handbook: Protocols across the spectrum. New York: WW Norton \& Company.

Shapiro, Robin (Ed.). 2009. EMDR solutions II: For depression, eating disorders, performance, and more. New York, NY: WW Norton \& Company.

Sterling, Peter. 2012. "Allostasis: a model of predictive regulation." Physiology \& behavior 106 (1):5-15. doi: 10.1016/j.physbeh.2011.06.004.

Sterling, Peter, and Joseph Eyer. 1988. "Allostasis: A new paradigm to explain arousal pathology." In Handbook of life stress, cognition and health., edited by Shirley Fisher and James Reason, 629-649. Oxford, England: John Wiley \& Sons.

Summerfield, Derek. 2001. "The invention of post-traumatic stress disorder and the social usefulness of a psychiatric category." Bmj 322 (7278):95-98.

Terr, L. C. 1991. "Childhood traumas: An outline and overview." American Journal of Psychiatry 148:10-20.

Ubl, Bettina, Christine Kuehner, Peter Kirsch, Michaela Ruttorf, Carsten Diener, and Herta Flor. 2015. "Altered neural reward and loss processing and prediction error signalling in depression." Social Cognitive and Affective Neuroscience 10 (8):1102-1112. doi: 10.1093/scan/nsu158.

Van der Kolk, Bessel A. 2005. "Developmental Trauma Disorder: Toward a rational diagnosis for children with complex trauma histories." Psychiatric annals 35 (5):401-408. doi: 10.3928/00485713-20050501-06. 
van der Kolk, Bessel A., and Onno van der Hart. 1989. "Pierre Janet and the breakdown of adaptation in psychological trauma." American Journal of Psychiatry 146 (12):15301540.

Weathers, Frank W, and Terence M Keane. 2007. "The Criterion A problem revisited: Controversies and challenges in defining and measuring psychological trauma." Journal of traumatic stress 20 (2):107-121. doi: 10.1002/jts.20210.

Wilkinson, Sam, Guy Dodgson, and Kevin Meares. 2017. "Predictive Processing and the Varieties of Psychological Trauma." Frontiers in Psychology 8 (1840). doi: 10.3389/fpsyg.2017.01840. 


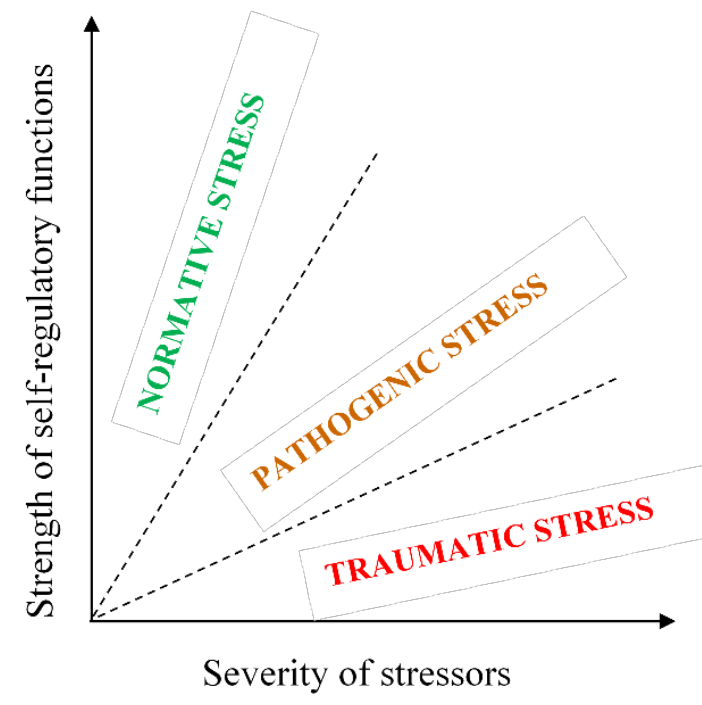

a

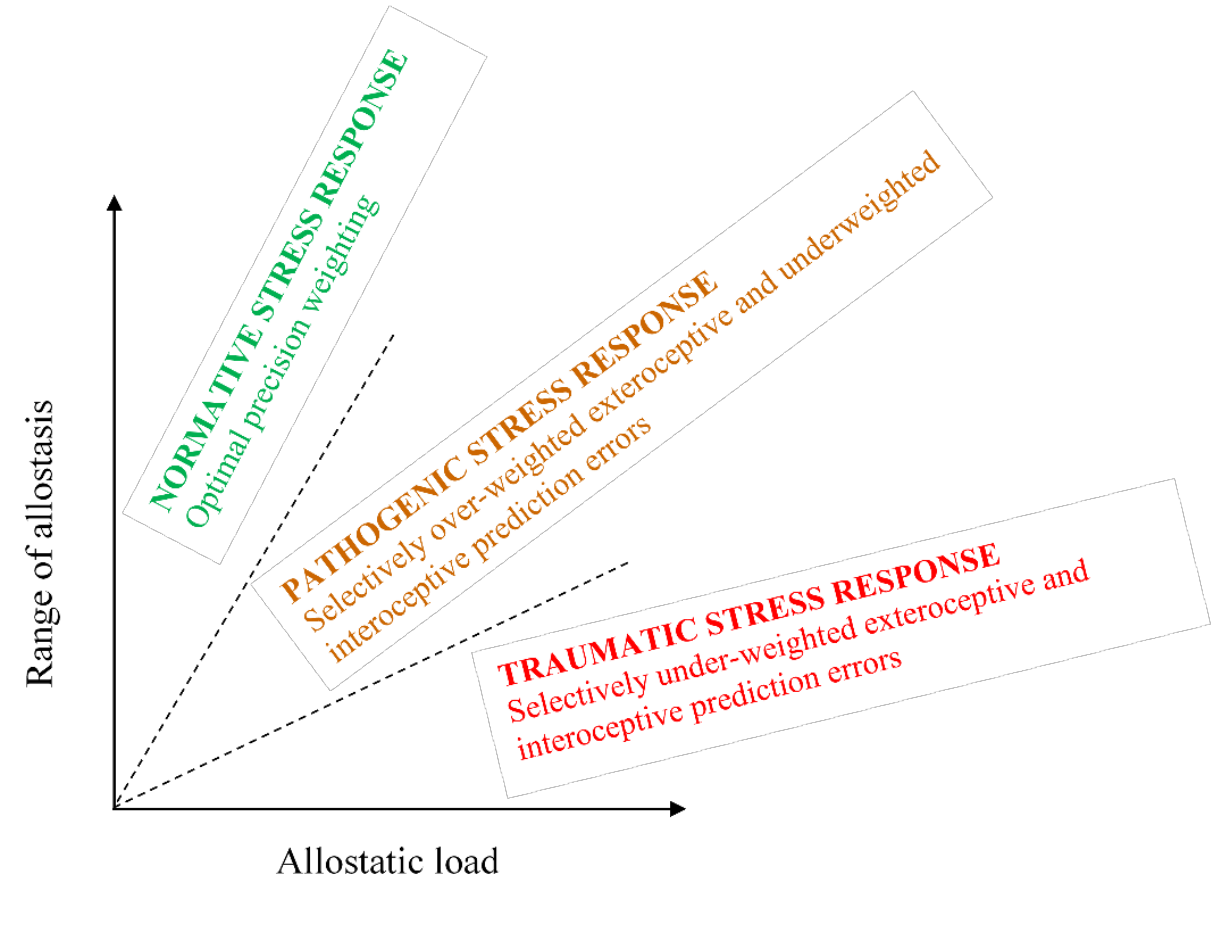

b

Figure 1. Hybrid categorical-dimensional model of stress (a) and stress response (b) continua (modified from Krupnik, 2019). 


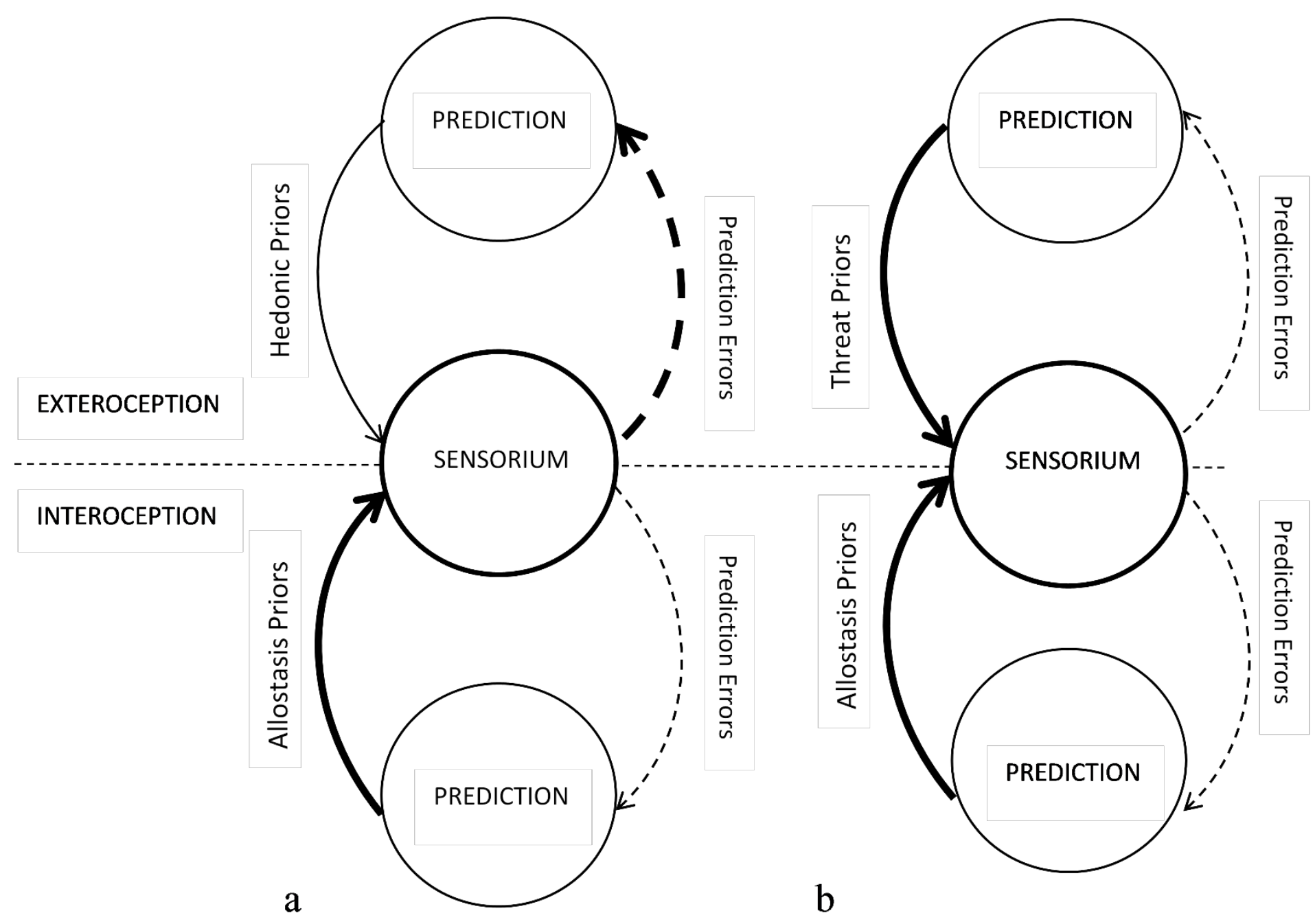

Figure 2. Imbalanced precision-weighting in pathogenic/depressive (a) and traumatic (b) stress response (modified from Krupnik 2020).

Arrow thickness denotes the relative precision. priors, - - - - prediction errors. 